\title{
Correlation between Cancer Research Trends and the Importance of Cancers based on Mortality and Diagnosis Rates: An Analysis of Altmetric Data
}

10.17975/sfj-2017-014

AUTHORS: Peter Chou ${ }^{1}$, Kevin Hong ${ }^{1}$, Chandler Lei ${ }^{1}$, Haolin Zhang ${ }^{1}$

SCHOOL: Earl Haig Secondary School, Toronto, Canada

\section{Abstract}

Aside from the infrequent news pertaining to medical breakthroughs or dangers to the public, medical research, especially in the field of cancer, is rarely discussed in depth. The public does not know the process in which specific fields of medical research receive funding, or how this funding is used to limit issues such as cancer. This study aims to provide clarity on cancer research trends. The amount of research papers pertaining to different types of cancers is compared against mortality and diagnosis rates to determine the amount of research attention given to a type of cancer, in relation to its effects on the general population.

Computational tools, such as Python, R, and Microsoft Excel, were used to analyze a dataset of research papers. Python was used to parse through JSON files and extract the abstract and Altmetric score of cancer research papers. $R$ was used to count the appearance of each type of cancer in the abstracts, and create histograms describing Altmetric scores and file frequency. Microsoft Excel was used to find correlations between Altmetrics' data and Canadian Cancer Society data, linking the amount of research to the impacts of cancer based on deaths and new cases.

The analysis from these tools revealed that breast cancer was the most researched cancer by a large margin, with nearly 1,700 papers, which is approximately four times the amount of the next leading type of cancer - prostate cancer. Although there were many research papers on the field of cancer, the Altmetric scores revealed that most of these papers did not gain significant online and media attention.

Comparing these results to Canadian Cancer Society data showed that breast cancer was receiving more research attention than the mortality and diagnosis rates suggest it should. There were four times more breast cancer research papers than the secondmost researched cancer, prostate cancer. This was despite the fact that breast cancer was fourth in mortality and third in new cases among all types of cancer. Inversely, lung cancer was underrepresented, with only 401 research papers, despite being the deadliest cancer in Canada.

\section{Key Words}

Cancer, Research, Treatment, Big Data, Altmetrics

\section{Introduction}

The focus of this paper is to determine which types of cancers are being researched the most, and whether the mortality and diagnosis rates have any impact on the number of research papers published. We hypothesize that deadlier and faster-growing cancers will have more research papers published about them.

This information could reveal the rate at which the scientific community is researching different types of cancers, and if the research rates are reflective of mortality and diagnosis rates. This can help scientists reconsider the funding that certain avenues of cancer research receive, and prompt further investigation to optimize research and identify which areas or research are being neglected.

This study investigates the correlations between the quantity and quality of research papers studying each type of cancer, and the dangers that these cancers pose to society. The quantity is measured by the amount of research papers that concern each type of cancer. The quality is measured through the Altmetric attention scores of these papers. The danger is measured through the number of new cases and deaths caused by each cancer in Canada during 2016.

The Altmetric attention score of a paper represents the attention a research paper receives through online sources. This includes social media sites, blogs, online newspapers and journals, and citations to the paper by other sources. The Altmetric attention score measures both quality and quantity of posts about the research papers, ranking the more read and more professionally-sourced posts higher than others; A post from a respectable newspaper, such as the Washington Post, would rank higher than blog and social media posts. 
import os import json import csv

os.chdir('directory') ourFiles = os.listdir()

for $i$ in ourFiles:

with open (i,'r') as f: try:

data $=$ json.load(f) except:

continue

with open('Results.csv','a',newline="') as file: try:

fields $=[\mathrm{i}, \operatorname{str}($ data['Altmetric_score' ['score']), str(data['selected_quotes'])]

writer $=$ csv.writer(file) writer.writerow(fields)

except: continue

Figure 1:

Code Sample displaying the creation of the CSV file containing the Altmetric score and abstract for each individual paper in Python.

\section{Materials and Methods}

A set of JSON (JavaScript Object Notation) files $(n=525,040)$ that contain cancer research papers was used to identify relevant papers over half a year. Of the information included in each JSON file, only the Altmetric scores and the abstracts were used. Using Python code, the information needed from these JSON files were transferred to one CSV file where every individual row represented information from a JSON file i.e. the program read files from a folder containing the JSON files, and wrote the Altmetric score and Abstract of each paper into individual rows of the CSV file.

Using $\mathrm{R}$, the abstracts were searched for mentions of different types of cancer, and the frequency of papers for each type of cancer was determined. The CSV file was loaded into an R program that was created to search for keywords in the abstract and to create graphs.

The data was then used to create histograms for each type of cancer, containing the number of the research papers in certain ranges of Altmetric scores. Using Microsoft Excel, Altmetrics' data was analyzed together with mortality and diagnostic data from the Canadian Cancer Society. These graphs displayed the relationships between different types of cancer, the frequency of research papers

freq $<-$ hist $(\log 10($ factor +1$)$, main $=$ names $($ histogram chart $[$ iter $])$, pertaining to each type of cancer, and the Altmetric score of the papers.

Data on the morality rates and diagnosis rates of cancers in Canada in 2016 was obtained from the Canadian Cancer Society and Statistics Canada, and compared against the number of research papers concerning each type of cancer. The data was moved to a CSV file where frequency - death graphs and frequency - new cases graphs were created through Excel.

\section{Results}

From the total 525,040 JSON files analyzed, 302,421 files contained both an abstract and an Altmetric score. 10,948 of these files were found to have "cancer" in their abstract. The number of files relating to individual types of cancers were counted and are displayed in Figure 3.

Data regarding the number of deaths and the number of new cases of cancer, obtained from the Canadian Cancer Society, is displayed in Figure 4.

Data regarding the Altmetric scores of papers of different types of cancer is displayed in Figure 5.

breaks $=10$,

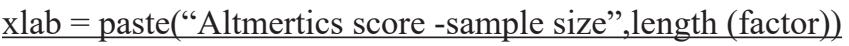

\section{Figure 2:}

Sample code showing the creation of histograms of the Altmetric scores of papers relating to each type of cancer. Scores are calculated by logs of 10. 
Type of Cancer

Cancer (total)

Breast

Prostate

Lung/Bronchial

Colorectal/Colon

Pancreatic

Pancreatic

Bladder

Leukemia

Esophageal/Esophagus

Lymphoma

Brain

Kidney/Renal

Stomach

Types of Cancer

Cancer (total)

Breast

Prostate

Lung/Bronchial

Colorectal/Colon

Pancreatic

Pancreatic

Bladder

Leukemia

Esophageal/Esophagus

Lymphoma

Brain

Kidney/Renal

Stomach
Number of JSON Files

\begin{tabular}{|c|}
\hline 10,948 \\
\hline 1,695 \\
\hline 428 \\
\hline 401 \\
\hline 315 \\
\hline 186 \\
\hline 164 \\
\hline 92 \\
\hline 88 \\
\hline 47 \\
\hline 40 \\
\hline 19 \\
\hline 7 \\
\hline
\end{tabular}

\section{Figure 3:}

Number of JSON files relating to the different types of cancer.

Figure 4: Number of deaths and new cases of different types of cancer in Canada during 2016. [1]

\section{Discussion}

The relationship between the frequency of JSON files on the type of cancer, and the number of deaths from each type of cancer in Canada during 2016 was graphed and is displayed in Figure 6. It was expected that a high number of deaths would correlate with a high frequency of research papers. This was generally true apart from two outliers.

Breast cancer papers were by far the most abundant, as there were nearly four times the amount of papers on breast cancer af- ter the next most frequent cancer: prostate cancer. Yet, the number of breast cancer deaths were the third highest among the cancers mentioned [1].

Lung cancer was the deadliest cancer in Canada in 2016, killing more than twice the amount of people compared to the second closest cancer: colorectal cancer [1]. However, the number of lung cancer papers ranked third among the types of cancer surveyed.

The relationship between the frequency of JSON files about each type of cancer and the number of new cases of each type of cancer 
in Canada during 2016 was graphed and is displayed in Figure 8. It was expected that a greater number of new cases would correlate with an increase of research paper frequency. This was generally true, except for one outlier.

While being a close third in the amount of new cases of each cancer in Canada during 2016 [1], there was nearly quadruple the amount of research papers published on breast cancer than the second most published cancer, prostate cancer, which has the third most amount of new cases.

These results show that breast cancer is receiving by far the most attention, despite not being the deadliest or the type of cancer with the greatest abundance of research done in 2016. Nearly the same amount of research papers has been published on breast cancer as the other 13 deadliest cancers in Canada combined. The number of research papers published appears to have a positive correlation with both the number of new cases and the number of deaths in 2016. However, breast cancer and lung cancer show that this is not always the case. Tobacco is known to cause lung cancer, and although heavy media coverage and educational programs have been produced detailing the risks, people continue to consume these products. Thus, little research is done, resulting in death and rates of new cases to remain high. Conversely, breast cancer is a cause behind numerous awareness and fundraising occasions, such as Breast Cancer Awareness month, that garners a
Types of Cancer

Breast

Prostate

Lung/Bronchial

Colorectal/Colon

Pancreatic

Pancreatic

Bladder

Leukemia

Esophageal/Esophagus

Lymphoma

Brain

Kidney/Renal

Stomach
Median Altmetric Score

1.25

3.35

1.75

2.00

1.50

2.30

1.25

1.75

1.50

3.50

13.65

6.45

3.20
Max Altmetric Score

$1,031.00$

632.6

618.90

1623.00

740.80

820.50

75.32

146.90

266.00

352.80

748.20

52.08

47.50

Figure 5: Altmetric scores of the research papers for different types of cancer

Frequency of Research Papers in Relation to Deaths Caused by Cancers

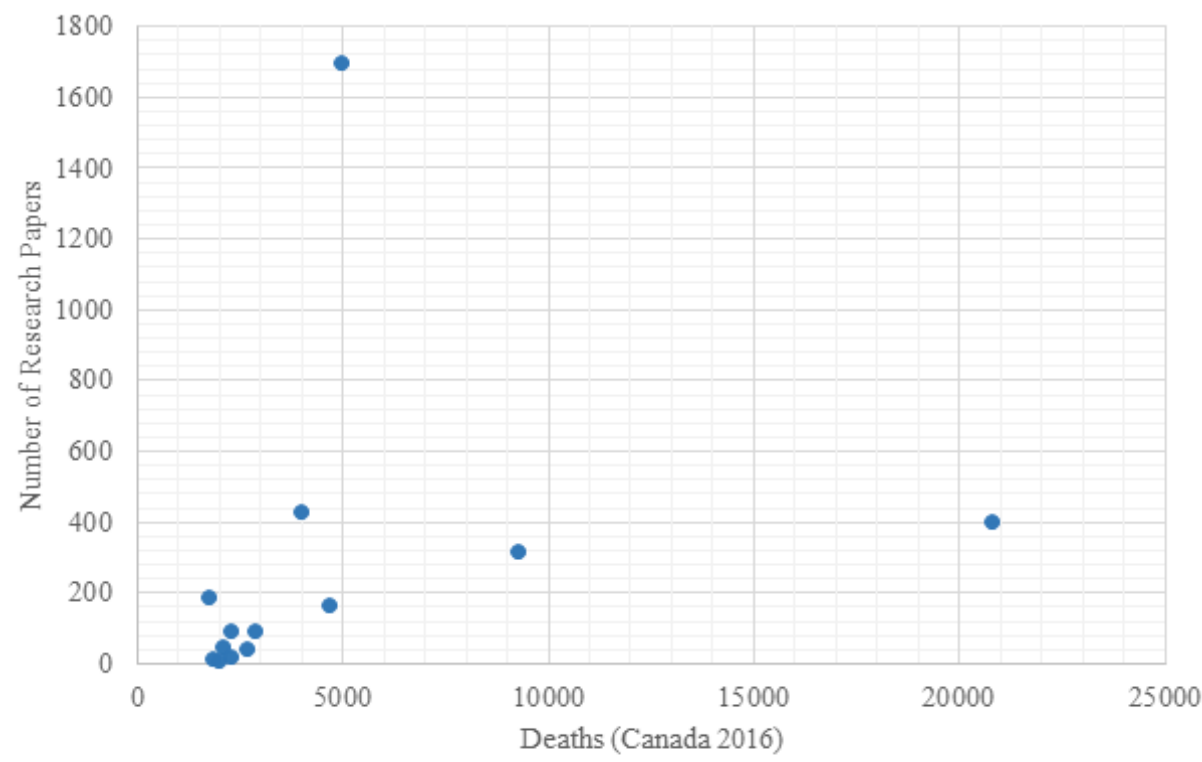

Figure 6:

Number of deaths of different types of cancer in Canada during 2016. [1] 
large amount of media attention and reaching professional sports leagues where NFL superstars wear pink to support breast cancer.

In addition to comparing the amount of research papers pertaining to each type of cancer, the amount of attention the papers received was compared through their Altmetric scores.

Histograms, examples included in Figure 8 and 9, were created for each type of cancer to compare the Altmetric scores and the frequency of papers with similar scores. Most of the cancer types resulted in the papers with a median Altmetric score between 1 and 4. However, kidney cancer and brain cancer papers had significantly larger median Altmetric scores. This suggests that the few papers that were related to kidney and brain cancer gained attention online. The Altmetric score data suggests that of the research papers published about cancers, few gain attention. The papers that are rarely read and referenced in news and social media retrieve low Altmetric scores. Of the cancers with more than 50 papers published, prostate cancer papers had the most evenly distributed Altmetric scores, while breast cancer had the least. Histograms of these scores are shown in figure 8 and 9 respectively.

Although breast cancer was recorded as the cancer type with the highest amount of research papers published, the papers have relatively low Altmetric scores, suggesting that they do not garner

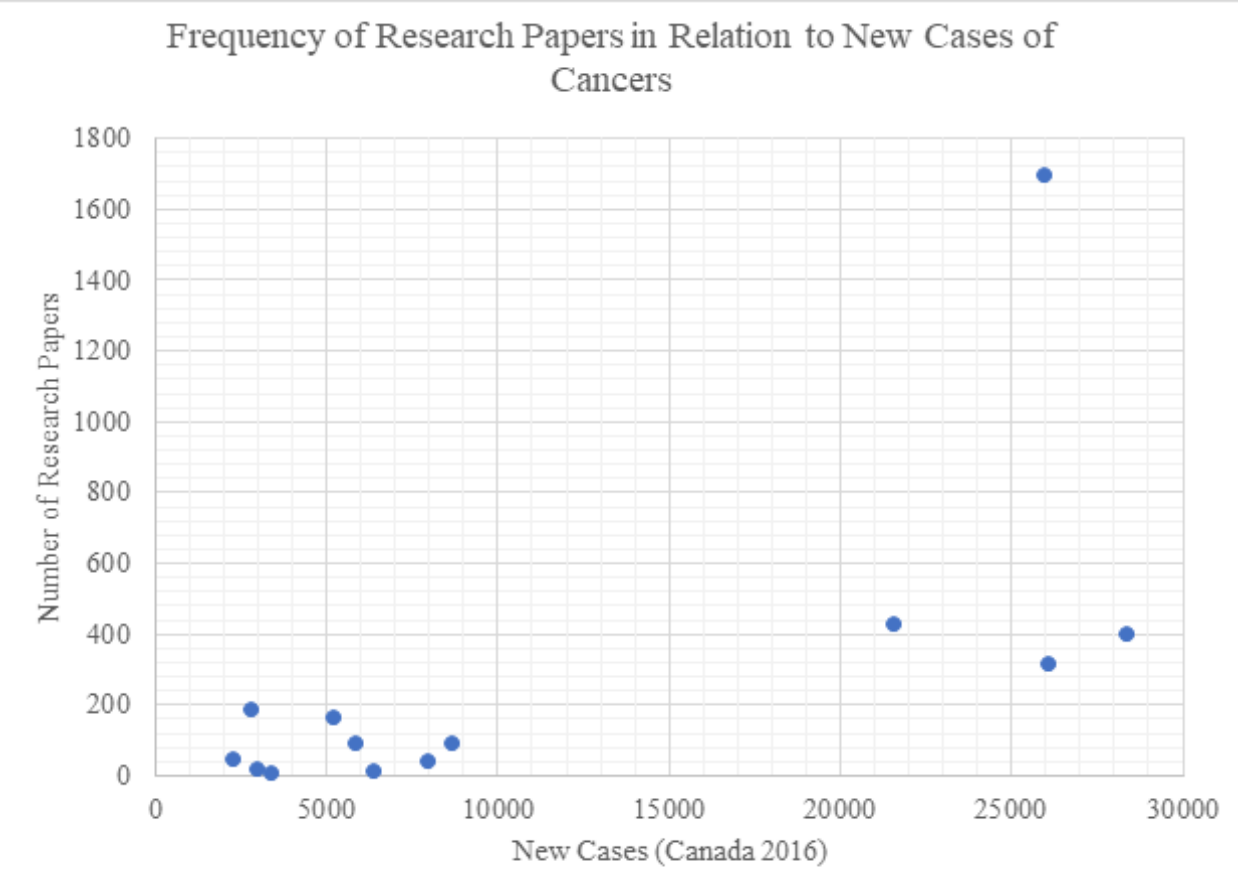

Figure 7:

Number of new cases of different types of cancer in Canada during 2016. [1]

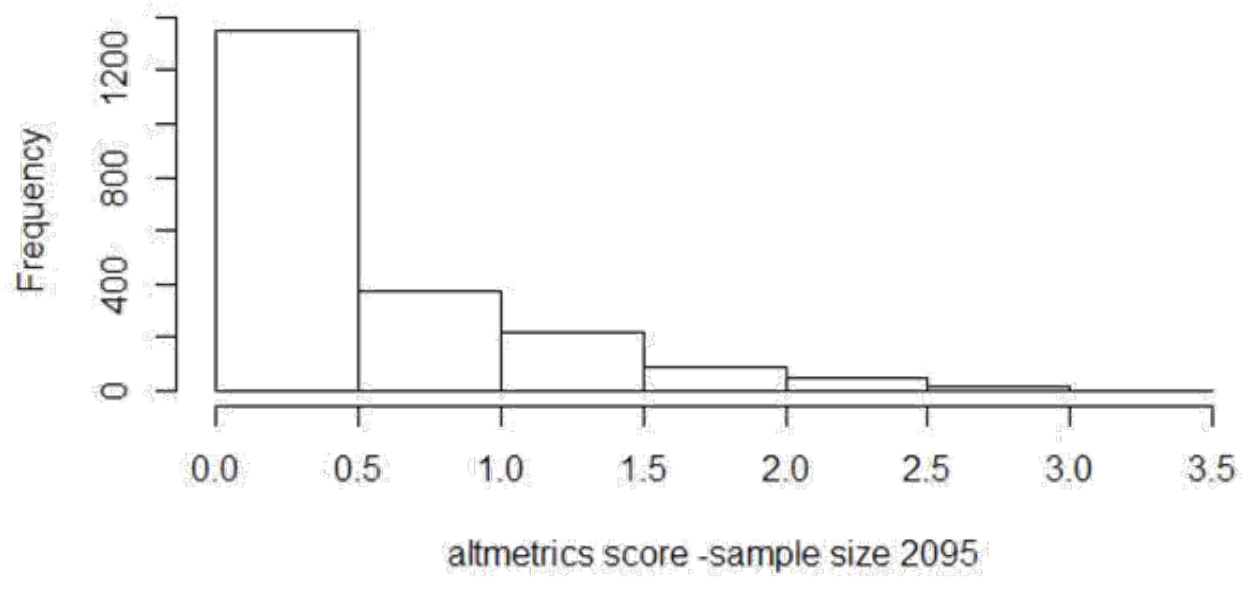

Figure 8: Altmetric score distribution of breast cancer papers. 


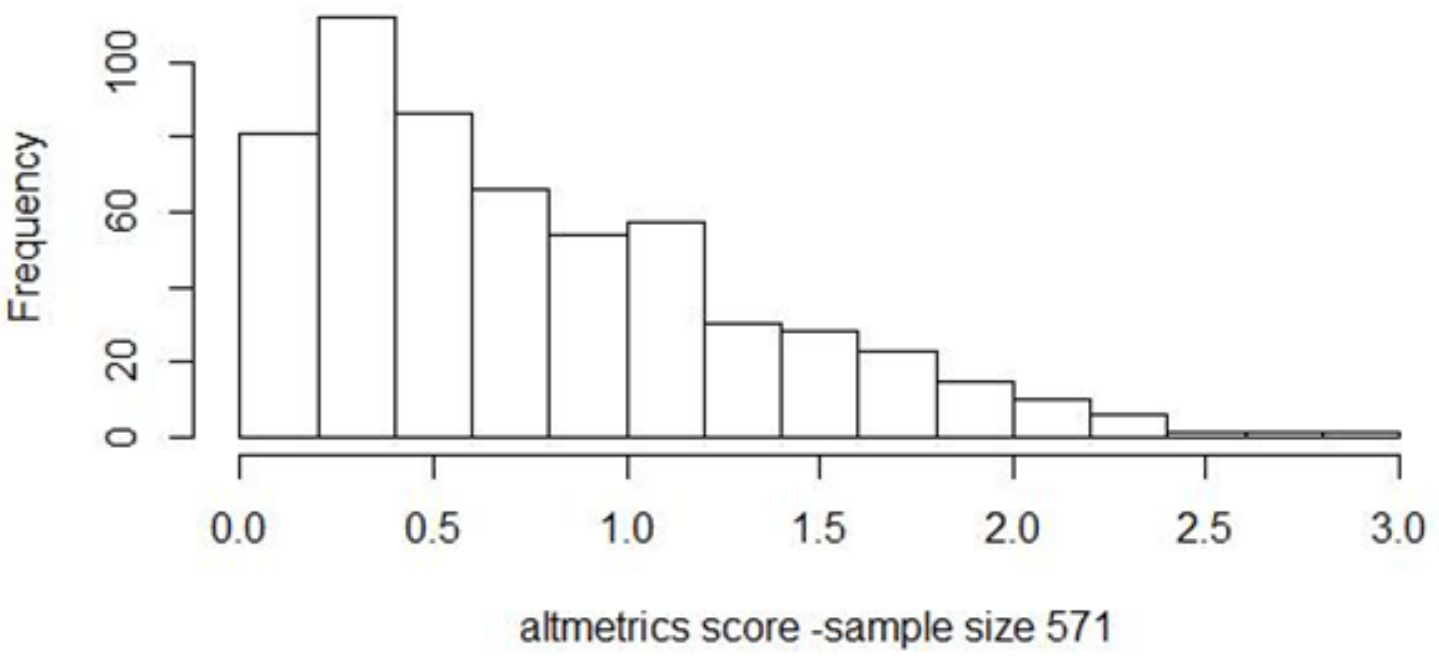

Figure 8: Altmetric score distribution of prostate cancer papers.

as much public attention as papers of other cancers. This could be due to the large number of papers being published, resulting in divided online attention or lack of public interest. There is a possibility that the research is too specific or scientific to garner the attention of the public, or that media outlets are not covering enough medical research.

A potential source of error is the omission of 200,000 files that did not contain both an Altmetric Score and abstract. These files could have contained research about cancer which could have altered our frequency counts.

\section{Conclusion}

This study revealed that the amount of research into specific types of cancer is generally reflective of their importance based on danger to the general population, with breast cancer and lung cancer being abnormalities. Breast cancer research has four times as many papers as the next most researched type of cancer, despite ranking third in mortality and diagnosis rates in Canada during 2016 [1]. However, because mortality and diagnosis rates, as well as the number of papers only apply for one year, the general trend cannot be observed. Lung cancer is more than four times deadlier than breast cancer, but the number of papers published is more than four times fewer than the number of papers published on breast cancer. Although mortality and diagnosis rates are not the only factors that determine the importance or prevalence of a type of cancer, they are good indicators of the potential optimization of funding. It is important to monitor the research rates of the different types of cancer, and compare them with the mortality and diagnosis rates when allocating research and funding.

\section{Acknowledgement}

We acknowledge our gratitude to Dr. Noukhovitch, our teacher, who has given us insight into our research.

\section{References}

[1] Canadian Cancer Society. Canadian cancer statistics 2016. Toronto: 2016 [cited 2017 December 21]. 27, 50 p. Available from: http://www.colorectalcancer.ca/IMG/pdf/Canadian-Cancer-Statistics-2016-EN.pdf 


\section{REVIEW}

\section{by Dr. Michael Duong}

\section{Director of Evidence Generation, Medical Affairs, Hoffmann-La Roche Limited}

In this paper, the authors studied the correlation that may or may not exist between the number of publications associated with various cancer types and their mortality and incidence rates. The authors propose that that cancers of high mortality or incidence are associated with a high number of publications which were determined by the mention of the cancer type in the published abstracts. Ultimately, the authors concluded that while there may be a trend towards increased frequency of publications with increased mortality and incidence rates of various cancer types, they were not able to find a correlation. Breast cancer was found to have highest number of publications by a wide margin, while only being the fourth highest in mortality. Similarly, lung cancer which has the highest mortality (by a wide margin) garnered significantly fewer publications and ranked 4th in frequency of publications. The authors further analyzed the "public interest" of these publications by evaluating the Altmetric Scores of the publications by cancer type. Overall, the authors concluded that there was limited attention given to cancer publications as most cancer types scored a median Altmetric Score between one and four.

This paper could have benefited from a more detailed description of the Altmetric Score, how it is determined and the relative value of the absolute score. It is stated that the scores overall are "relatively low" but we do not know how this compares to publications in other fields of research. Overall, the hypothesis is an interesting one to test but the result is not unexpected. It would stand to reason that frequency of publication in a given cancer type would directly translate to the level of research activity. This would then translate to significant advances in treatment which would ultimately lead to better outcomes and lower mortality rates. This is clearly evident in the authors' findings within breast cancer. This field has benefited from an enormous amount of research and as such, many diagnoses (e.g. Her2 positive breast cancer) which would have had the worst prognosis 10 years ago, now have one of the best prognoses in cancer. An interesting follow-up analysis to this study could be to evaluate this correlation longitudinally, over time. This could demonstrate that dynamic growth in publication frequency over time could be correlated to better mortality results, which may be attributed to increased research activity. Clearly this study has - as all research should - inspired this reader to ask additional questions and propose additional studies that could advance our knowledge and understanding of the particular question. A commendable job by the researchers and authors of this paper. 